\title{
Key Application Technologies of High Efficiency Power Quality Control Systems
}

\author{
Ding-Guo Liu ${ }^{*}$, Zhi-kang Shuai ${ }^{\dagger}$, Chun-ming Tu ${ }^{*}$, Ying Cheng ${ }^{* *}$, and An Luo* \\ $\dagger^{\dagger}$ College of Electrical and Information Engineering, Hunan University, Changsha, China \\ ${ }^{* *}$ Maintenance Company, Hunan Electric Power Corporation, Changsha, China
}

\begin{abstract}
Large capacity reactive power compensation and harmonic control in the low-voltage grid of an enterprise, are important technical means to improve power quality and reduce power loss. In this paper, the principle of an efficient power quality controller is analyzed. Then, key application technologies of the HPQC which would influence the performances of the HPQC are studied. Based on an analysis of the harmonic shunt problem, a frequency dividing control strategy of the HPQC continuous subsystem is proposed. A parameter design method of the HPQC discrete subsystem and its installation method are also proposed to ensure the system compensation effect. HPQC systems have been designed for a copper foil plant. The effectiveness of this paper has been verified by the simulation and application results.
\end{abstract}

Key words: High efficiency power quality control, Harmonic circulation, Dividing-frequency control strategy, Parameters design method, Structural design method

\section{INTRODUCTION}

The development of power supplies plays an important role in the social economy. In recent years, due to the increase in the non-linear load in the grid, for example, rectifiers and electric railways, power quality problems are getting more and more serious. To resolve these problems, a variety of power quality control techniques have emerged [1]-[3].

In high-voltage grids, the more widely used harmonic control and reactive power compensation devices are as follows: passive power filters (PPF), active power filters (APF), hybrid active power filters (HAPF) and Static Var Compensators (SVC), etc [4]-[7]. Due to the constraints of voltage level and capacity, it is difficult for APFs to be widely used in high voltage grids. HAPFs can achieve high-capacity static reactive power compensation and harmonic dynamic elimination, but they cannot adjust reactive power continuously. SVCs have good performance in large capacity dynamic reactive power compensation, but in the dynamic regulation process, SVCs produce harmonics, even when a passive power

Manuscript received Jun. 18, 2012; revised Feb. 4, 2013

Recommended for publication by Associate Editor Kyeon Hur.

${ }^{\dagger}$ Corresponding Author: zhikangshuai@hotmail.com

Tel: +86-731-8882-3441, Fax: +86-731-8882-3441, Hunan University

*Coll. of Electrical and Information Eng, Hunan University, China

** Maintenance Company, Hunan Electric Power Corporation, China filter device is deployed, the filtering effect is unsatisfactory [9], [10]. Therefore, the function of most power quality control systems is relatively simple, and only reacts on one aspect of power quality, thus restricting the improvement and implementation of energy saving in the grids.

In low-voltage grids, with the growth of nonlinear loads, the low power factor and harmonic pollution lead to a mass of loss, and the supply voltage can hardly meet the demands of end users. Compared to high-voltage girds, the reactive power and harmonic current in low-voltage systems is larger. The main technical tools for harmonic control and reactive compensation in low-voltage grids (380V side) are as follows: fixed compensation capacitors (FC), contactors automatically switching capacitors, thyristor switched capacitors (TSC), thyristor switched filters (TSF), static synchronous reactive power compensators (with filtering function), etc [11], [12]. Thyristor controlled capacitors can achieve reactive power step-adjustment, but they do not have filtering capability. Thyristor controlled switching filters have the functions of reactive power step-adjustment and filtering, but they can not realize continuous adjustment of the reactive power. Static synchronous reactive power compensators possess the functions of dynamic reactive power compensation and harmonic suppression, voltage fluctuations and flicker suppression, imbalance compensation, etc. but due to the constraints of power electronic devices' capacity and cost, it is 
difficult to use them in large-scale projects.

To improve the performance of power quality control systems, high efficiency power quality control technologies have received more attention. The subject of high efficiency power quality control includes the following aspects: (1) High efficiency power quality control systems should have a better control effect in terms of a variety of power quality problems, not just one particular problem. (2) High efficiency power quality control systems should be able to achieve high-performance in the case of lower-cost devices. (3) High efficiency power quality control is also reflected in the rational use of existing power quality controllers. (4) High efficiency power quality control is a global concept, all of the power quality control systems should be considered from a global point of view, thus they should be able to achieve an optimal control effect with a lower investment. References [13] and [14] proposed high efficiency power quality control systems (HPQC) for high-voltage and low-voltage grids, and the topological structures as well as the control methods were studied.

To realize high efficiency power quality control of a large-scale mining enterprise, a high efficiency power quality control system is proposed in this paper. In Section II, the principles of the HPQC are analyzed; In Section III, key technologies of the HPQC, including the dividing-frequency control strategy, the parameter design method and the structural design method are proposed; In Section IV, a high efficiency power quality control method is designed for a mining enterprise, and the HPQC devices significantly improve the product quality and the energy saving effect.

\section{OPERATION MECHANISM OF HPQC}

In [14] a dynamic hybrid Var compensator (HVC) composed of multi-group TSCs in parallel with a DSTATCOM is proposed, as shown in Fig. 1(a), which can be regulated continuously between its maximum capacitive power and its inductive reactive power. When the group numbers of TSCs vary, the HVC can regulate continuously between its maximum capacitive reactive power and its maximum inductive reactive power. The discrete sub-system TSC of the HVC system can supply high capacity reactive power, and it has a low cost and is easy to control. The capacity of the DSTATCOM is small, but it plays an important role in dynamic reactive power compensation and voltage flicker suppression. Therefore, the HVC system has combined the advantages of the TSC and the DSTATCOM.

However, when a distribution grid contains nonlinear loads, because its equivalent impedance is inductive, the TSC will result in harmonic amplification. This will result in grid instability, and ultimately lead to capacitor breakdown. Fig.1 (b) shows the situation of harmonic amplification when the HVC capacitor switch is on and off. The z-axis is the multiple of the harmonic amplification, the $\mathrm{x}$-axis is the harmonic order (2nd

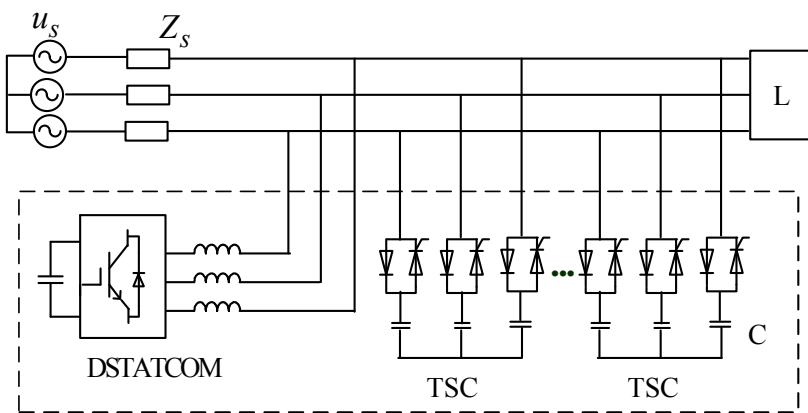

(a)

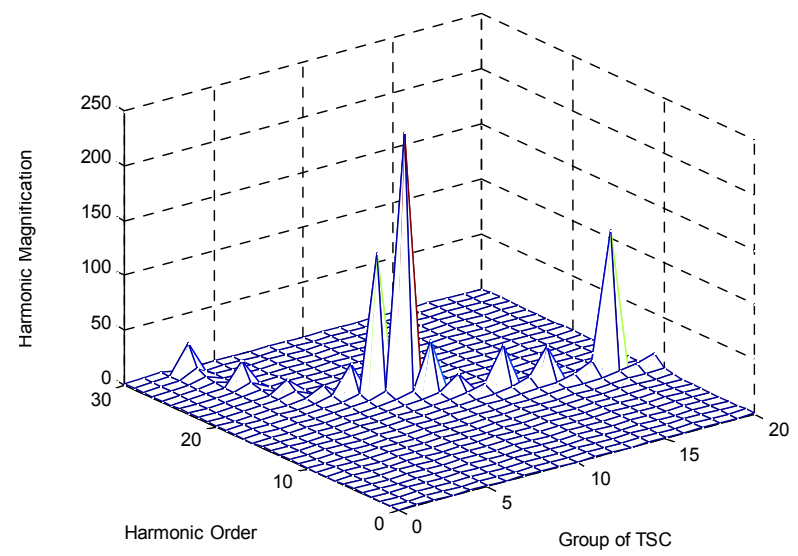

(b)

Fig.1 Topology (a) and Harmonic magnification characteristic (b) of HVC.

-30th harmonics), and the y-axis is the number of times the capacitor turns on. From Fig.1 (b), when the TSC is put into operation, there is a harmonic amplification between the TSC and the grid, which is not conducive to long-term reliable operation of the system.

Therefore, in this paper, the DSTATCOM in Fig. 1(a) generates both reactive power and harmonics. The discrete subsystem TSC is replaced by a TSF. The new topology can be used for large capacity reactive power compensation and harmonic elimination. As a result, the continuous subsystem DSTATCOM and the discrete subsystem TSF make up the high efficiency power quality control system (HPQC). The topology of the HPQC system is shown in Fig. 1(a). The harmonic amplification characteristic of the HPQC is shown in Fig. 2(b). It can be seen that it is similar to that of the HVC. The TSF is composed of the 5th and 11th TSF. From Fig. 2(b), it can be seen that that the harmonic amplification phenomena is restrained.

\section{KeY APPLiCATION TECHNOLOGIES OF HPQC}

HPQC has good performance in reactive power compensation and harmonic elimination, especially when the harmonics and the reactive power are in large capacities. However, if the design method of the system is unsuitable, 


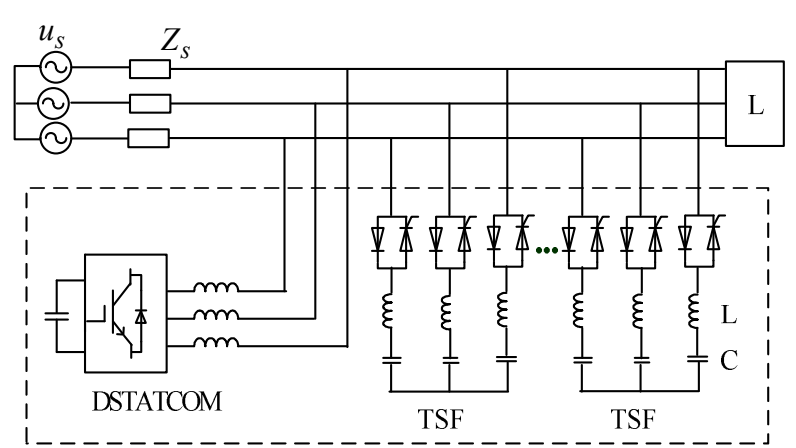

(a)

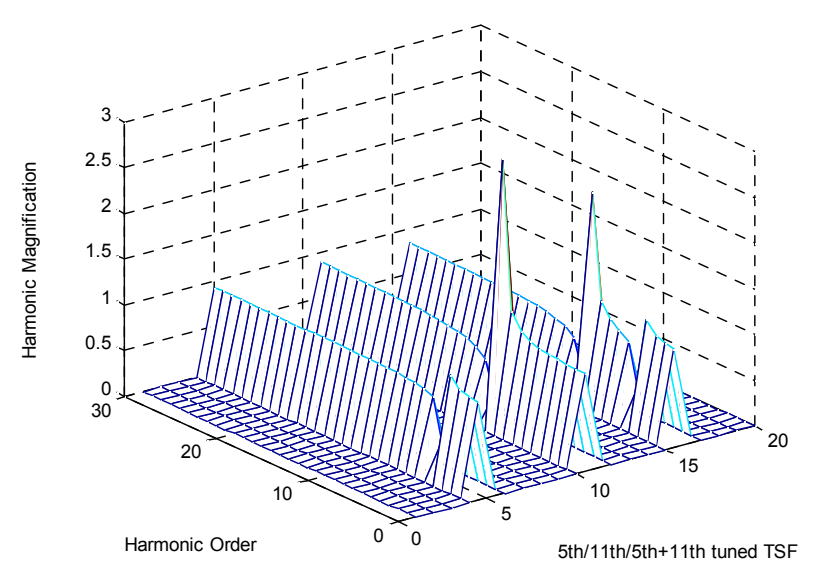

(b)

Fig. 2. Topology (a) and Harmonic magnification characteristic (b) of HPQC.

harmonic circulation may be formed, and the performance of the system will be influenced. In this section, to improve the performance of the HPQC, the key technologies of the HPQC are analyzed, such as the control strategy of the HPQC, the parameter design method, and the structural design method of the discrete subsystem of the HPQC.

\section{A. Dividing-frequency Control Strategy of HPQC}

For the output harmonic currents of the HPQC continuous subsystem, the discrete subsystem of the HPQC also will provide harmonic channels. Therefore, part of the harmonic current output by the HPQC continuous subsystem flows to the grid, thereby reducing the supply current distortion; and part of it flows to the HPQC discrete subsystem. The split ratio is determined by the parameters of the HPQC discrete subsystem and the equivalent impedance of the grid. As a result, if the strategy is unsuitable, harmonic circulation between the discrete subsystem and the continuous subsystem may be formed, which will seriously influence the performance of the HPQC system. In this section, a dividing-frequency control strategy is proposed to restrain the harmonic circulation.

The electrical model of the HPQC is shown in Fig.3 (a),

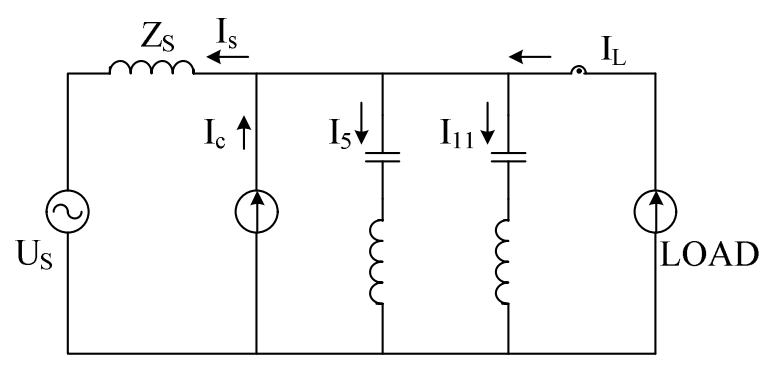

(a)

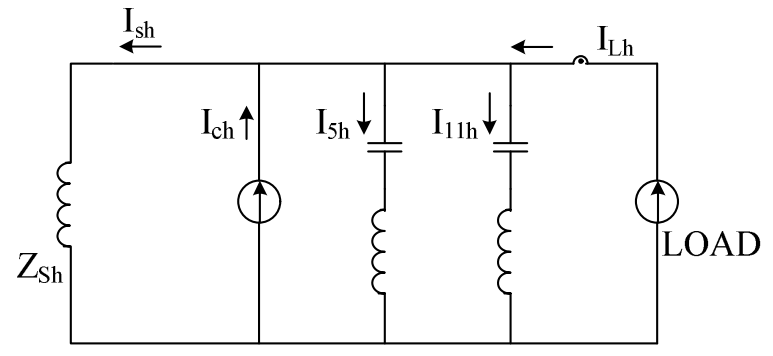

(b)

Fig. 3. Electrical mode (a) and Electrical model in harmonic domain (b) of HPQC.

where the passive branches of the HPQC discrete subsystem are tuned at the $5^{\text {th }}$ and $11^{\text {th }}$ order harmonic, respectively. In Fig.3 (a), $I_{c}$ is the output current of the HPQC continuous subsystem, $I_{L}$ is the load current, $U_{S}$ is the grid voltage, $I_{5}$ and $I_{11}$ are the branch currents passing through the $5^{\text {th }}$ order and the $11^{\text {th }}$ order discrete subsystems, respectively. When only considering the influence of harmonic currents, the equivalent electrical model of the HPQC system is shown in Fig.3 (b).

From Fig.3 (b), it can be seen that the output current of the continuous subsystem split-flows through its two streaming channels, that is, the discrete subsystem and the grid. It can also be seen that the shunt condition of the harmonic current that closes to the discrete subsystem tuning frequency is particularly evident. If the capacity of the continuous subsystem is large enough, it will track the detected load harmonic current as much as possible. At this point, if the frequency of the harmonic currents output by the HPQC continuous subsystem is the same as the tuning frequency of the discrete subsystem, there will be a larger part of the harmonic currents flowing into the discrete subsystems of the HPQC. The specific work process is as follows:

Assuming the filtering coefficient of the discrete subsystem's $n^{\text {th }}$ harmonic current is $\eta$, that is, if the $n^{\text {th }}$ load harmonic current is $x$ ampere, then the $n^{\text {th }}$ harmonic current that flows into the discrete subsystem of the HPQC can be expressed as $y$.

$$
y=\eta * x
$$

Similarly, for the continuous subsystem of the HPQC, there is also $\eta$ times the amount of the $\mathrm{n}^{\text {th }}$ harmonic current 
flowing into the discrete subsystem. Take the $5^{\text {th }}$ harmonic for example, assuming that the $5^{\text {th }}$ load harmonic is $\mathrm{M}$ ampere, the filter coefficient $\eta$ of the discrete subsystem is 0.5 , ignoring the influence of the continuous subsystem, the amount of $5^{\text {th }}$ harmonic current flowing into the grid is:

$$
I_{T S F 5}=\eta^{*} M=0.5 M
$$

For the continuous subsystem, its reference signal is:

$$
I_{\text {STAT }}^{*}=G_{P} * M=-M
$$

Ideally, the output current of the continuous subsystem is:

$$
I_{S T A T}=-M
$$

Part of the output current of the continuous subsystem flows into the discrete subsystem and part of it flows into the grid. The $5^{\text {th }}$ harmonic current, which is output by the continuous subsystem of the HPQC, flowing into the grid is:

$$
I_{S 5}^{\prime}=(1-\eta) *(-M)=-0.5 M
$$

The $5^{\text {th }}$ harmonic current, which is output by the continuous subsystem of the HPQC, flowing into the discrete subsystem is

$$
I_{T S F 5}^{\prime}=\eta^{*}(-M)=-0.5 M
$$

According to Thevenin's superposition theorem, when the HPQC is put into operation, the supply harmonic currents and the harmonic currents flowing into the discrete subsystem are:

$$
\begin{aligned}
& I_{S h}=I_{S 5}+I_{S 5}^{\prime}=0 \\
& I_{T S F h}=I_{T S F 5}^{\prime}+I_{T S F 5}=0
\end{aligned}
$$

From equations (7) and (8), it can be seen that for the frequency that is the same as what the discrete subsystem is tuned at, when the continuous subsystem is completely tracking the reference signal, harmonic elimination can be realized. However, the harmonic current flowing into the discrete subsystem is zero at this time, and the discrete subsystem could not achieve the filtering effect.

When the continuous subsystem is limited by the capacity and can not track the load harmonic current, take the $5^{\text {th }}$ harmonic for example, assuming the $5^{\text {th }}$ output harmonic current of the continuous subsystem is $\mathrm{N}(\mathrm{N}<\mathrm{M})$, where:

$$
N=\delta * M
$$

At this point, the current generated by the continuous subsystem flowing into the grid is:

$$
I_{S 5}^{\prime \prime}=(1-\eta) *(-\delta * M)=-0.5 * \delta * M
$$

The current generated by the continuous subsystem flowing into the discrete subsystem is:

$$
I_{T S F 5}^{\prime \prime}=\eta^{*}\left(-\delta^{*} M\right)=-0.5 * \delta * M
$$

The supply harmonic current and the harmonic current flowing into the discrete subsystem of the HPQC are:

$$
\begin{aligned}
& I_{S h}=I_{S 5}+I_{S 5}^{\prime \prime}=0.5 *(1-\delta) * M \\
& I_{T S F h}=I_{T S F 5}^{\prime \prime}+I_{T S F 5}=0.5 *(1-\delta) * M
\end{aligned}
$$

It can be seen that when the continuous subsystem can not fully track the load harmonic current, the HPQC cannot eliminate the harmonics completely.

Therefore, if the continuous subsystem generates the same harmonic as what the discrete subsystem is tuned at, a huge harmonic circulation will be formed between the continuous and discrete subsystems and the performance of the subsystem will be degraded. Therefore, a dividing-frequency control strategy is presented. That is, the discrete subsystem tunes at the main character harmonics (as described in the text, the $5^{\text {th }}$ and $11^{\text {th }}$ ), and provides grading adjustable reactive power capacity. The continuous subsystem only manages a single harmonic or a few other order harmonics (as described in this paper, the $7^{\text {th }}, 13^{\text {th }}, 17^{\text {th }}, 19^{\text {th }}$ harmonics). Therefore, the requirement for the capacity of the continuous subsystem is relatively small, and dynamic reactive power compensation can also be achieved. When the proposed dividing-frequency control strategy is adopted, the harmonic circulation phenomenon will be almost negligible.

\section{B. Parameters Design Method of the Discrete Subsystem}

A number of effective methods for single-tuned passive power filter parameter optimization design have been proposed, including the cost-optimal method, multi-objective genetic algorithm optimization, etc. However, these optimization algorithms rarely focus on some specific problems in engineering applications, such as the harmonic amplification problems of single-tuned passive filters. These problems usually influence the performance of filters, and sometimes even play a decisive role. In this section a new parameters design method is proposed, and the harmonic amplification problem in particular is considered.

The $\mathrm{N}^{\text {th }}$ harmonic impedance of a single-tuned passive power filter is:

$$
Z_{f n}=R_{f n}+j\left(n \omega_{s} L-\frac{1}{n \omega_{s} C}\right)
$$

where, $\omega_{S}$ is the fundamental frequency and $R_{f n}$ is the equivalent inner resistance of the filter reactor.

The resonance frequency of the passive power filter is:

$$
f_{n}=\frac{1}{2 \pi \sqrt{L C}}
$$

After determining the resonance frequency of the single-tuned passive power filter, the parameters design of the filter capacitor $\mathrm{C}$ and the filter reactor $\mathrm{L}$ are mainly influenced by the following:

(1) The resonance frequency and the number of groups of single-tuned passive power filters.

Resonance frequency and the number of groups of single-tuned passive power filters are decided by the load harmonic condition. For certain non-linear devices, the characteristic harmonics can be estimated. For example, the characteristic harmonics of a piece of 6-pulse rectifier equipment are primarily the $5^{\text {th }}$ and $7^{\text {th }}$, and sometimes it is 
necessary to consider the $11^{\text {th }}$ and $13^{\text {th }}$. Therefore, for 6-pulse-based non-linear loads, a $5^{\text {th }}$ and $7^{\text {th }}$ single-tuned passive power filter can be chosen. For 12 pulse-based non-linear loads, an $11^{\text {th }}$ and $13^{\text {th }}$ single-tuned passive power filter can be chosen.

(2) The reactive power compensation capacity allocation method of single-tuned passive power filter groups.

The total reactive power compensation capacity of a passive power filter is demanded by the load reactive power, and multiple sets of the filter capacity are distributed by the harmonic content ratio. Thus the effect of the harmonic filter and the filter component selection will be taken into account.

(3) The values of filter capacitor $\mathrm{C}$ and filter reactor $\mathrm{L}$. The value of the filter capacitor can be calculated according to the reactive power compensation capacity of the passive power filters.

$$
C=\frac{Q_{c}}{\omega_{s} * U^{2}}
$$

where, $U$ is the effective value of the line-voltage of the grid.

The value of the filter reactor can be obtained according to formula (16).

$$
L=\frac{1}{C n^{2} \omega_{S}^{2}}
$$

(4) The quality factor $Q$ of the filter reactor.

The filter reactor quality factor is:

$$
Q=\frac{\omega_{n} L}{R_{f n}}=\frac{1}{\omega_{n} R_{f n} C}
$$

The equivalent impedance of a single-tuned filter in the resonance frequency is inversely proportional to its $\mathrm{Q}$. The greater $\mathrm{Q}$ is, the smaller the impedance is, and the better the frequency selectivity is. However, the filter itself is more sensitive to system parameters changes with an increasing $\mathrm{Q}$. Meanwhile, if the filter groups resonate with the grid equivalent impedance when $\mathrm{Q}$ is too large, the degree of harmonic amplification will be greater. Therefore, the quality factor values should be considered carefully. The following method can be used to determine the optimum $Q$ value:

$$
Q=\frac{\cos e+1}{2 t \sin e}
$$

where, $e$ is the greatest impedance angle of the system and $t$ is the greatest frequency offset of the system.

After obtaining the quality factor values according to equation (16), it is necessary to verify that the reactor temperature can meet the requirements for designing the filter reactor. If $\mathrm{Q}$ is low, the reactor temperature may be too high when the system runs, which will threaten the long-term reliability of the system.

(5) The installed capacity of the single-tuned passive power filter.

The installed capacity of a single-tuned passive power filter will directly determine the overall cost of the device and the component selection. In the design of a single-tuned filter, the filter component values is related to the filtering effect. However, it is only a part of the design work. Designing components to withstand voltage and current is of the same importance. If a defective design causes a poor ability to withstand voltage and current, it will affect the system's long-term reliable operation, while a high value selection will lead to a substantial cost increase. Single-tuned passive power filter capacitors and reactors are mainly influenced by the fundamental frequency voltage and the grid tuning frequency currents. Therefore, the filter capacitor voltage under normal operation is:

$$
U_{c}=U_{s}+\frac{i_{n}}{n \omega_{s} C}
$$

where, $U_{s}$ is the supply voltage and $i_{n}$ is the $\mathrm{n}^{\text {th }}$ harmonic current filtered by the filter.

In consideration of the most extreme case, which is where the peak of the fundamental voltage and the $\mathrm{n}^{\text {th }}$ harmonic voltage just overlap. Therefore, in this paper, the choice of the filter capacitor withstand voltage value is the algebraic sum of the harmonic voltage in the capacitor generated by the fundamental voltage and the harmonic current. The installed capacity of the filter capacitor is:

$$
Q_{c} \approx 3 * U_{c}^{2} \omega_{s} C
$$

For the filter reactor, the main considerations of the parameter design are the reactor voltage level, the reactor inductance and the rated current. The reactor inductance can be calculated from equation (17), and the rated current value is codetermined by the reactive power compensation current and the filtered harmonic current, that is:

$$
i=\sqrt{i_{s}^{2}+i_{n}^{2}}
$$

where, $i_{S}$ is the reactive power compensation current.

Therefore, the capacity of the filter reactor is:

$$
Q_{L}=i_{s}^{2} \omega_{s} L+i_{n}^{2} n \omega_{s} L
$$

(6) The parameter adjustment of the filter groups.

A PPF is usually composed of a capacitor and a reactor, and the equivalent impedance of the grid is mainly an inductor. As a result, the harmonic amplification phenomenon may occur, which can result in the grid becoming unstable, cause the capacitor to withstand a high voltage, reduce the capacitor life, or even damage the capacitor. Therefore, it is necessary to consider the harmonic amplification characteristic between the compensation system and the grid when designing the parameters of a PPF.

An electrical model of the load harmonic current amplification analysis is shown in Fig.4. In this model, $i_{n}$ is the $\mathrm{n}^{\text {th }}$ harmonic current, $X_{S}$ is the equivalent reactance of the grid, $X_{C}$ is the capacitance of the single-tuned filter capacitor, and $\mathrm{X}_{\mathrm{L}}$ is the reactance of the single-tuned filter reactor $\left(\mathrm{X}_{\mathrm{L}}=\right.$ $\mathrm{AX}_{\mathrm{C}}, \mathrm{A}$ is the reactance ratio). Defining the ratio of the 


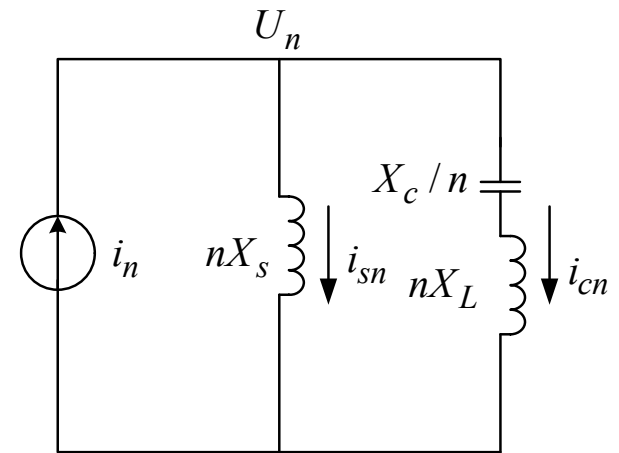

Fig. 4. Electrical model of load harmonic current amplification analysis.

harmonic voltage before and after the single-tuned filter is adopted as a harmonic voltage magnification $\left(F_{V n}\right)$. From Fig.4 it can be seen that:

$$
F_{V n}=\left|\frac{n^{2} A-1}{n^{2}(S+A)-1}\right|
$$

where, $S=X_{\mathrm{S}} / X_{\mathrm{C}}$.

Assuming the load harmonic current contains the $\mathrm{m}^{\text {th }}$ order Assume that the load harmonic current contains the $\mathrm{m}^{\text {th }}$ order, which is lower than the series resonant frequency tuned by the PPF. For the $\mathrm{m}^{\text {th }}$ order harmonic current, the single-tuned filter is capacitive, while the equivalent impedance of the grid is inductive. As a result, when the single-tuned filter is used, the $\mathrm{m}^{\text {th }}$ harmonic voltage of the gird will increase (that is to say, the $\mathrm{m}^{\text {th }}$ harmonic voltage is enlarged), and the security of grid is threatened.

Therefore, it is necessary to analyze the harmonics amplification circumstances when designing the parameters of the filters. If the harmonic amplification circumstance is within the design requirements, and the design parameters are reasonable. If the characteristic harmonics magnification situation is serious, the design parameters should be adjusted. The adjustment principle is to make the filter groups capacity depart from its resonance limit capacity under the premise of ensuring the total amount of reactive power compensation and the filtering effect. After several adjustments, a reasonable set of filter parameters can finally be determined.

\section{Structural Design Method of Passive Power Filters}

Experience shows that even if the filter parameters are well designed, if the filter reactors are placed irrationally and cause severe mutual coupling, the filtering effect in each phase will be inconsistent, and the overall filter effect may be reduced by $10 \%-20 \%$. So the structure of the filter reactor should be designed in reasonable ways to eliminate the adverse effects caused by reactor coupling.

There are three common installation mode of the filter reactor, including three-phase stacked, three-phase horizontal straight arrayed and three-phase horizontal triangular

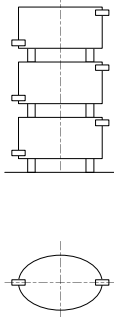

(a)

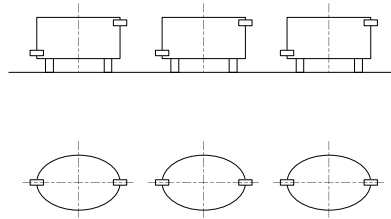

(b)

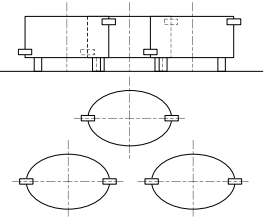

(c)
Fig. 5. Basic installation, mode three-phase stacked (a), horizontal straight arrayed (b), and horizontal triangular arrangement (c) of the three-phase reactor

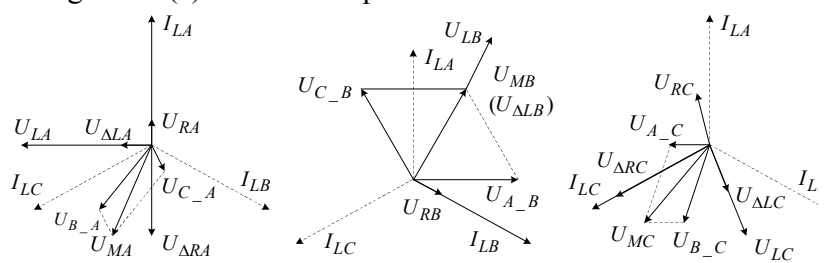

(a)

(b)

(c)

Fig. 6. Vector diagrams of phase-A (a), phase-B (b) and phase-C (c) when reactors are connected in three-phase stacked mode

arrangement, as shown in Fig.5.

When the reactors of the filter are placed in the form of three-phase stacked, the floor space is the smallest, which is suitable for indoor display and in a wide range of applications. Assuming the current flowing through the three-phase filter reactor is equivalent and that the phase follows by a difference of 120 degrees, the vertical distance between the $\mathrm{A}$, $\mathrm{B}$ phase and the $\mathrm{B}, \mathrm{C}$ phase reactor is the same. In order to reduce the tension on the insulator, the winding of the B-phase reactor is generally reverse connected to the loop with the A phase and the $\mathrm{C}$ phase reactor. In other words, the winding direction of the $\mathrm{B}$ phase reactor is contrary to the $\mathrm{A}$ and $\mathrm{C}$ phase reactor winding. Then the reactor voltage and the current vector diagrams of the A, B, C phase are shown in Fig.6.

where, $U_{\Delta L A}$ and $U_{\triangle R A}$ are the projection of $U_{M A}$ in the direction of $U_{L A}$ and $U_{R A}$, respectively; $U_{\Delta L B}$ and $U_{\triangle R B}$ are the projection of $U_{M B}$ in the direction of $U_{L B}$ and $U_{R B}$, respectively; $U_{\triangle L C}$ and $U_{\triangle R C}$ are the projection of $U_{M C}$ in the direction of $U_{L C}$ and $U_{R C}$, respectively.

Fig. 6 shows that the mutual inductance of the filtering reactor makes the following changes in the equivalent reactance and the equivalent resistance of the three-phase reactor:

(1) With the mutual influence of the inductors of phase B and phase $\mathrm{C}$, the equivalent reactance of the A-phase filter inductor increases, while the equivalent resistance of the A-phase reactor changes in the direction of its inherent resistance to reduction. Fig.6 (a) shows that $U_{\triangle L B}$ and 
${ }_{C_{-} A}^{I_{L A}}$

(a)

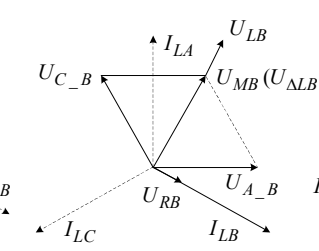

(b)

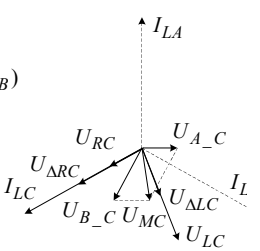

(c)
Fig. 7. Vector diagrams of phase-A (a), phase-B (b) and phase-C (c) when reactors are connected in horizontal straight arrayed mode.

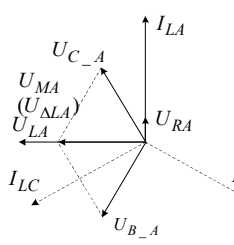

(a)

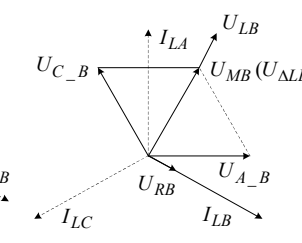

(b)

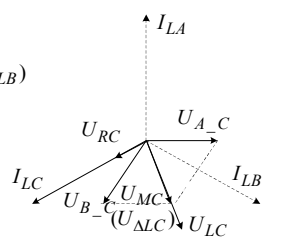

(c)
Fig. 8. Vector diagrams of phase-A (a), phase-B (b) and phase-C (c) when reactors are connected in horizontal triangular arrangement mode.

$U_{L B}$ are in the same direction, and $U_{\Delta L B}$ and $U_{M B}$ reverse.

(2) With the mutual inductor of the inductors of phase A and phase $\mathrm{C}$, the equivalent reactance of the B-phase filter inductor significantly increases, while the equivalent resistance of the B-phase reactor remains unchanged. Fig.6 (b) shows that $U_{\triangle L B}$ and $U_{L B}$ are in the same direction, and $U_{\triangle L B}$ and $U_{M B}$ overlap.

(3) With the mutual influence of the inductors of phase A and phase $\mathrm{B}$, the equivalent reactance of the $\mathrm{C}$-phase filter inductor increases, while the equivalent resistance of the B-phase inductor also increases. Fig.6 (c) shows that $U_{\Delta L C}$ and $U_{L C}$ are in the same direction, and $U_{\Delta R C}$ and $U_{R C}$ reverse.

It can be seen that when the three-phase filter inductors are placed in the stacked manner, and the B-phase inductor winding is in a reverse connection, the equivalent reactance of the three-phase reactor increases, thus saving materials and reducing the filter reactor volume. However, under the influence of the mutual inductance, the equivalent resistance of the A phase and $\mathrm{C}$ phase reactors increases, which makes the equivalent quality factors of the two reactors reduce, which affects the performance of PPF. In addition, it is difficult to keep the three-phase harmonic filter rate constant.

When the horizontal straight arrayed mode is adopted, the three-phase filter reactors are in the same horizontal plane. At this point, the mutual inductance of the three-phase reactor is almost the same as the state of the three-phase stacked. However, the mutual inductance has the opposite sign because the magnetic field of the other two-phase reactor is opposite to that in the state of the three-phase stacked. Placed

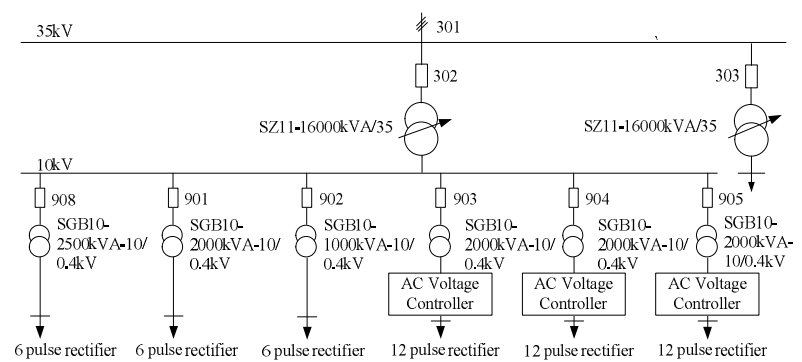

Fig. 9. The distribution network schema of a copper plant.

in the form of the horizontal straight arrayed, the three-phase filter reactor mutual inductance vector diagrams are shown in Fig.7.

It can be seen from Fig.7 that by the influence of the mutual inductance, the equivalent reactance of Phase-A increases, while the equivalent resistance of Phase-A changes in the direction of its inherent resistance to reduce. In addition, the reactor of Phase-B significantly increases, while the equivalent resistance of Phase-B remains basically unchanged. Furthermore, both the equivalent reactance and the equivalent resistance of Phase-C increase.

In the horizontal triangular arrangement mode, the three-phase reactors of the single-tuned passive power filter are located in an equilateral triangle with a horizontal plane. At this point, the mutual inductance vector diagrams between the reactors are shown in Fig.8.

Fig. 8 shows that if the parameters of the inductors are the same and in strict accordance with the mode of the standard triangular arrangement, the three-phase mutual inductance is the same. If the currents flowing through the three-phase reactor are equal to each other and 120 degrees of phase difference exist, the equivalent reactance of the three-phase increases due to the impact of the mutual inductance, which helps to reduce the reactor material and size. Meanwhile, with the influence of the mutual inductance, the equivalent resistance of the three phases is essentially the same, and the resistance is unaffected by the impact of the mutual inductance. As a result, the horizontal triangular arrangement mode is an ideal method for inhibiting the adverse influence of mutual inductance. Therefore, the horizontal triangular arrangement mode is adopted is this paper.

\section{SIMULATION AND APPLICATION RESUltS}

\section{A. Application Background}

The electrical equipment of a copper plant in Hefei is mainly rectifier devices. The electric power of the copper is supplied by a $35 \mathrm{kV}$ substation. The model of the main

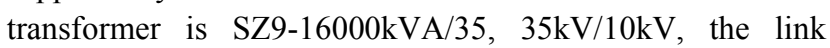
group is Ynd11, and the impedance voltage is $8 \%$. A diagram of the grid is shown in Fig.9, where, the $35 \mathrm{kV}$ transformer capacity of the short circuit is $645 \mathrm{MVA}$, and that of the $10 \mathrm{kV}$ 
transformer is 247MVA.

As is shown in Fig.9, there are six different capacity $10 \mathrm{kV}$ transformers to supply power for the different types of rectifiers. These include the 6-pulse controlled rectifier, the high-frequency rectifier, and the 12-pulse rectifier power supply.

The characteristic harmonics caused by the load carried by transformers I-III are the $5^{\text {th }}, 7^{\text {th }}, 11^{\text {th }}$ and $13^{\text {th }}$ with a total harmonic distortion (THD) of $30 \%$. A large number of harmonic currents flowing through the rectifier transformer will lead to a transformer temperature rise as well as numerous energy losses. Consequently, reliable operation of the transformer is threatened. On the other hand, harmonic currents affect the normal operation of copper foil equipment and it can even lead to product failure. The characteristic harmonics caused by the load under transformers IV-VI are the $11^{\text {th }}$ and $13^{\text {th }}$. Since transformers IV-VI form a 36 pulse rectifier system, only transformers I-III are considered.

In this paper, a low-voltage HPQC is adopted in the low-voltage side of transformers I-III for large capacity harmonic control and reactive power dynamic adjustment. The low-voltage side of transformers IV-VI do not have a special harmonic control. The high-voltage HPQC system is installed in the $10 \mathrm{kV}$-side to govern the harmonics and the reactive power produced by transformers IV-VI as well as I-III.

Since the characteristic harmonics current is as high as several hundred amperes, the HPQC consists of the $5^{\text {th }}$ and $11^{\text {th }}$ passive power filters and an APF. The $5^{\text {th }}$ and $11^{\text {th }}$ characteristic harmonic currents are filtered by the $5^{\text {th }}$ and $11^{\text {th }}$ passive filters, while the resonant frequency of $5^{\text {th }}$ and $11^{\text {th }}$ passive filters are far away. This effectively avoids the influence of the $5^{\text {th }}$ passive filter filtering effect caused by the $11^{\text {th }}$ passive filter, and it ensures the filtering effect of the passive filters. The continuous subsystem dynamically outputs reactive power according to the load fluctuations and achieves continuous adjustment of the reactive power as well as dynamic control of the $7^{\text {th }}$ and other harmonics. In this case, the HPQC can obtain a better effect for both the reactive power compensation and harmonic treatment. It can also save investment and have a low cost.

\section{B. Simulation Results}

Simulation results of the 6-pulse controlled rectifier connected to transformer I are shown in Fig.10. In this figure, I_La and I_sa are the load harmonic current and the supply harmonic current, respectively.

It can be seen from Fig.10, that the THD in the $400 \mathrm{~V}$-side of transformer I decreases from $35.2 \%$ to $14.5 \%$ after the HPQC is adopted. This is due to the fact that the HPQC system mainly governs the $5^{\text {th }}, 7^{\text {th }}$ and $11^{\text {th }}$ characteristic harmonics. The load harmonic current is not completely filtered by the limitations of the passive power filter itself and

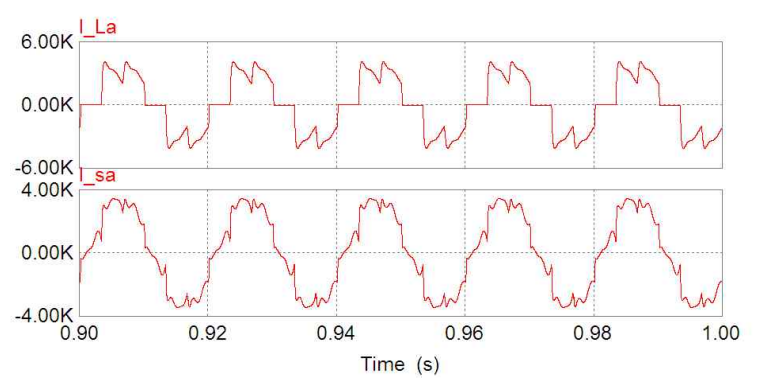

Fig. 10. Simulation result of the 6-pulse controlled rectifier connected to transformer I when $\alpha=30^{\circ}$

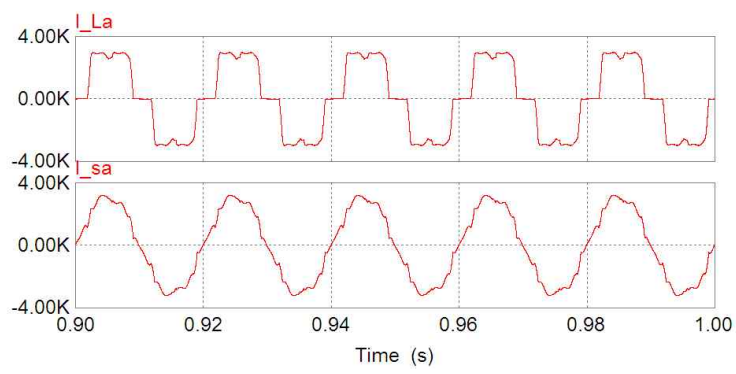

(a)

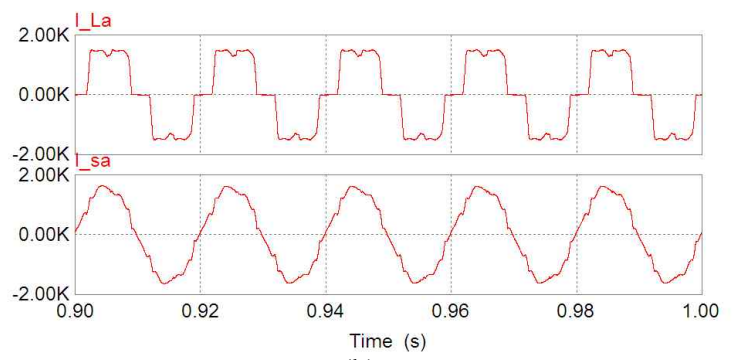

(b)

Fig. 11. Simulation results of high frequency switch rectifiers connected to transformers II (a) and III (b)

the impact of the load harmonic current. However, the harmonic content has been significantly reduced, and the power factor increases from 0.82 before compensation to 0.95 .

Simulation results of the high frequency switch rectifiers connected to transformers II and III are shown in Fig. 11.

It can be seen from the waveforms of the supply current before and after the HPQC is adopted, that the grid current distortion rate in the $400 \mathrm{~V}$-side of transformer II decreases from $30 \%$ to $8.1 \%$, and power factor increases to 0.95 . The grid current distortion rate in the $400 \mathrm{~V}$-side of transformer III decreases from $30 \%$ to $8.9 \%$, and power factor increases to 0.95 .

\section{Application Results}

The developed HPQC systems have been in successful operation in a copper plant, Fig.12 shows the application results of the HPQC. 

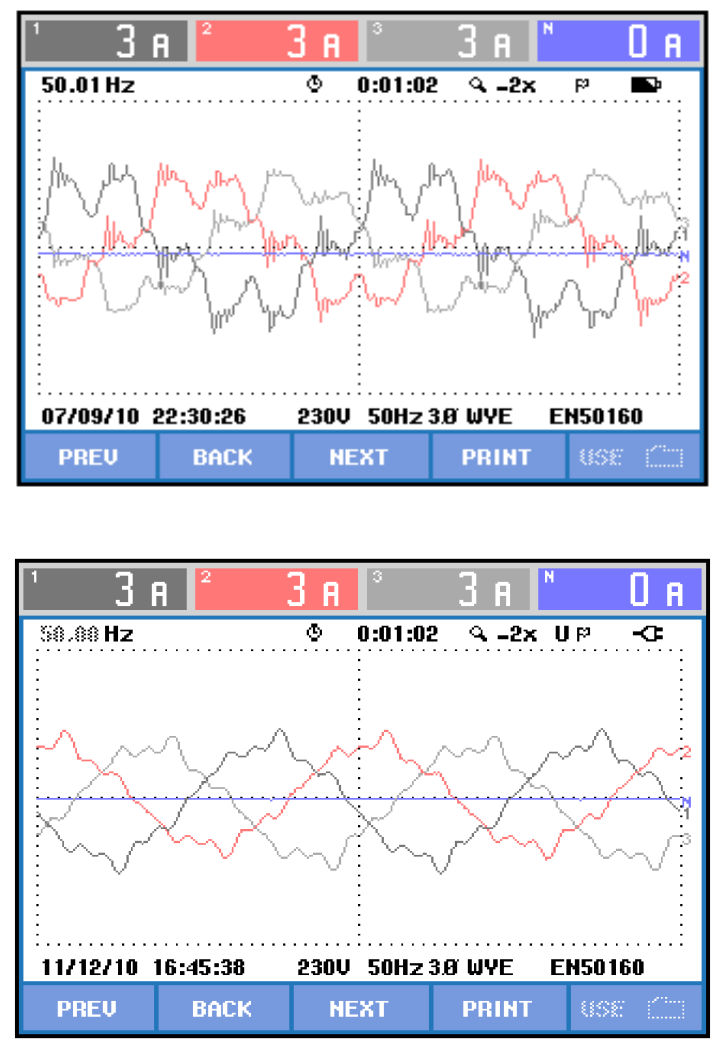

(a)
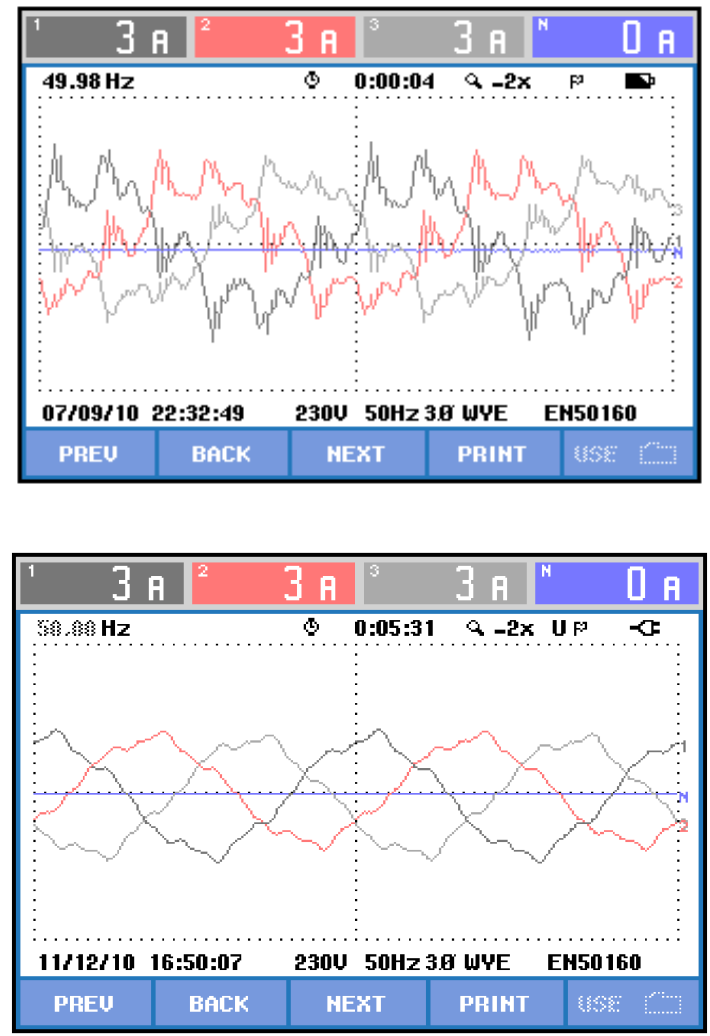

(b)
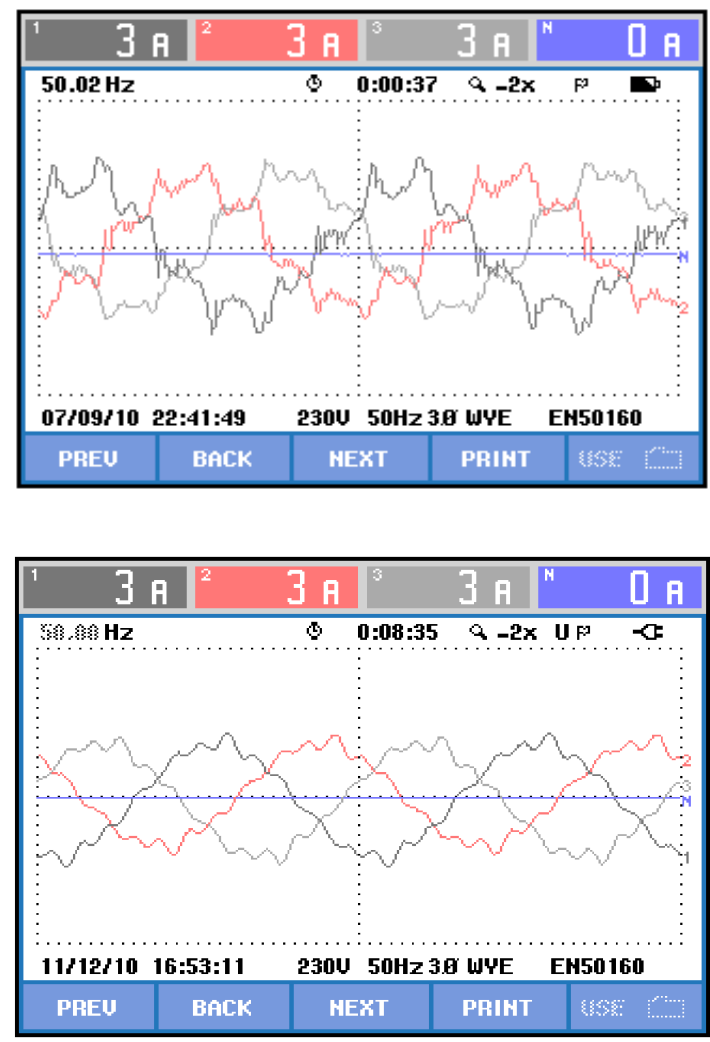

(c)

Fig. 12. Supply current under transformer I (a), transformer II (b), and transformer III (c) before and after HPQC are adopted

Fig. 12 shows that the distortion rate of the supply current is high before the HPQC is adopted. The THDs of the supply current in the $400 \mathrm{~V}$-side of transformers I-III are $36.8 \%$, $38.4 \%$ and $33.2 \%$, respectively. When the HPQC systems are adopted, the THDs of the supply currents of transformers I-III are decreased to $4.8 \%, 5.1 \%$ and $4.4 \%$, respectively. In particular the $5^{\text {th }}, 7^{\text {th }}, 11^{\text {th }}$ and $13^{\text {th }}$ characteristic harmonics are significantly reduced. At the same time, the power factor has also been increased form 0.89 to 0.96 . Therefore, the proposed HPQC system has good performance in harmonic elimination and reactive power compensation.

\section{CONCLUSIONS}

A HPQC system is proposed in this paper, which can realize large capacity reactive power compensation and harmonic control. The key application technologies of the HPQC system, such as the frequency dividing control strategy of the HPQC continuous subsystem, the parameter design method of the HPQC discrete subsystem, as well as its installation method, are presented. Based on the proposed key application technologies, a HPQC system is designed for a copper foil plant. Simulation and application results have verified the effectiveness of this system. 


\section{ACKNOWLEDGMENT}

This work was supported by the National Natural Science Foundation of China (No.51207048, No. 51277060); The New Teacher Fund for Doctor Station, the Ministry of Education (No. 20120161120011); The plan for the growth of young teachers, Hunan University.

\section{REFERENCES}

[1] W. Shireen and L. Tao, "A DSP-based active power filter for low voltage distribution systems," IEEE Trans. Power Electro., Vol. 78, No.9, pp. 1561-1567, Sep. 2008.

[2] O. Vodyakho and C. C. Mi, "Three-level inverter-based shunt active power filter in three-phase three-wire and four-wire systems," IEEE Trans. Power Electron., Vol. 24, No.5, pp. 1350-1363, May 2009.

[3] S. S. Patnaik and A. K. Panda "Comparison of current harmonic compensation capability of APF with two control strategies in 3-phase 4-wire system employing Bacterial foraging optimization," India Conference (INDICON), 2011 Annual IEEE, pp.1-6, 2011.

[4] P. Dang, J. Petzoldt, and T. Ellinger, "Stability analysis of multi-parallel APF systems," Power Electronics and Applications (EPE 2011), Proceedings of the 2011-14th European Conference on, pp.1-8, 2011.

[5] S. Fukuda and R. Imamura, "Application of a sinusoidal internal model to current control of three-phase utility-interface converters," IEEE Trans. Ind. Electron., Vol. 52, No. 2, pp.420-426, Apr. 2005.

[6] A. Khaligh, J. R. Wells, P. L. Chapman, and P. T. Krein, "Dead-time distortion in generalized selective harmonic control," IEEE Trans. Power Electron., Vol. 23, No. 3, pp. 1511-1517, May. 2009.

[7] G. K. Singh, A. K. Singh, and R. Mitra, "A simple fuzzy logic based robust active power filter for harmonics minimization under random load variation," Electric Power Systems Research, Vol. 77, No. 8, pp. 1101-1111, Jun. 2007.

[8] D. Lijie, L. Yang, and M. Yiqun, "Comparison of high capacity SVC and STATCOM in real power grid," Intelligent Computation Technology and Automation (ICICTA), 2010 International Conference on., Vol. 1, pp. 993-997, 2010.

[9] K. M. Son, K. S. Moon, S. K. Lee, and J. K. Park, "Coordination of an SVC with a ULTC reserving compensation margin for emergency control," IEEE Trans. Power Del., Vol. 15, No. 4, pp. 1193-1198, Jul. 2000.

[10] D. A. N. Jacobson and R. W. Menzies, "Comparison of thyristor switched capacitor and voltage source GTO inverter type compensators for single phase feeders," IEEE Trans. Power Del., Vol. 7, No. 2, pp. 776 -781, Mar. 1992.

[11] Z. Jianhua, D. Guanping, X. Gang, Z. Jie, Z. Hui, and W. Shuying, "Design of the control system for thyristor switched capacitor devices," Transmission and Distribution Conference and Exposition, 2003 IEEE PES. Vol. 2, pp.606-610, 2003.

[12] A. D. Le Roux, H. D. T. Mouton, and H. Akagi, "DFT-Based repetitive control of a series active filter integrated with a 12-pulse diode rectifier," IEEE Trans. Power Electron., Vol. 24, No. 6, pp. 1515-1521, Jun. 2009.
[13] A. Luo, Z. Shuai, W. Zhu, and Z. J. Shen, "Combined system for harmonic suppression and reactive power compensation," IEEE Trans. Ind. Electron., Vol. 56, No.2, pp. 418-428, Feb. 2009.

[14] Z. Shuai, A. Luo, Z. J. Shen, W. Zhu, Z. Lv, and C. Wu, "A dynamic hybrid var compensator and a two-level collaborative optimization compensation method," IEEE Trans. Power Electron., Vol. 24 No. 9, pp. 2091-2100, Sep. 2009.

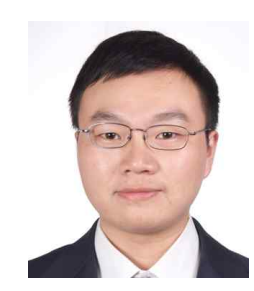

Ding-Guo Liu was born in Hunan, China, on June 6, 1979. He received his B.S. and M.S. from the College of Electrical and Information Engineering, Hunan University, Changsha, China, in 2005 and 2008, respectively. Since 2009 he has been a doctorate student in the College of Electrical and Information Engineering, Hunan University. His current research interests include harmonics suppression, reactive power compensation and smart grids.
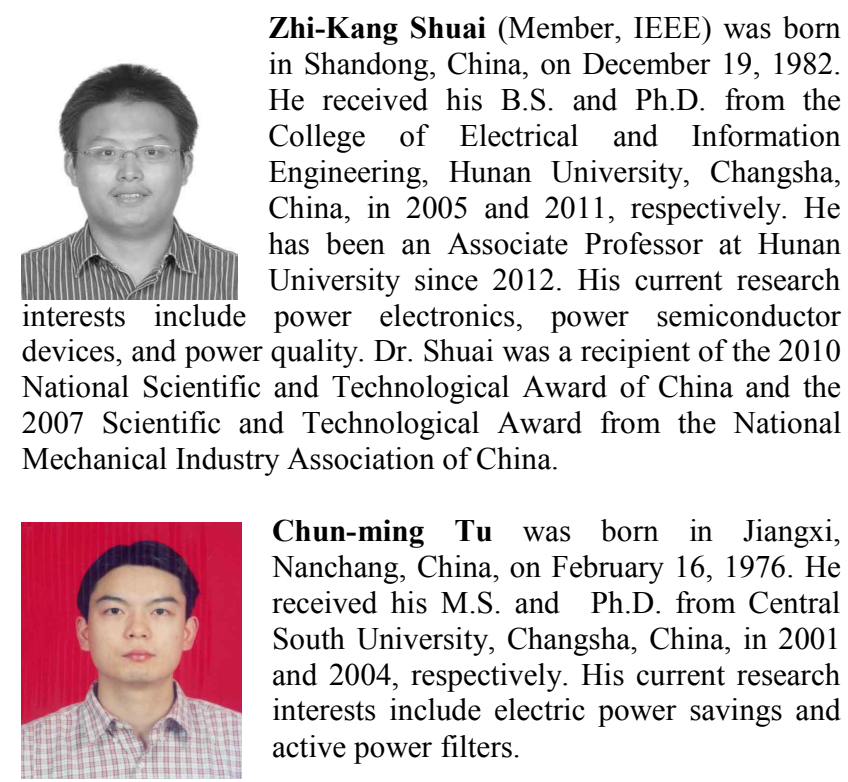

Chun-ming Tu was born in Jiangxi, Nanchang, China, on February 16, 1976. He received his M.S. and Ph.D. from Central South University, Changsha, China, in 2001 and 2004, respectively. His current research interests include electric power savings and active power filters.

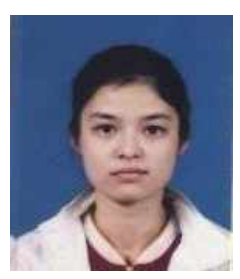

Ying Cheng was born in Hunan, Changsha, China, on May 17, 1985. She received her B.S. form the College of Electrical Engineering, Southwest Jiaotong University, Chengdu, China, in 2007, and her M.S. from the College of Electrical and Information Engineering, Hunan University, Changsha, China, in 2010. Her current research interests include power electronics and power quality.

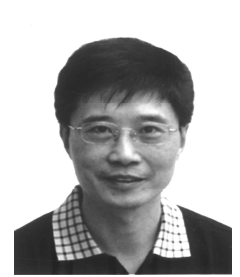

An Luo (Senior Member, IEEE) was born in Changsha, China, on July 21, 1957. He received his B.S. and M.S. from Hunan University, Changsha, China, in 1982 and 1986, respectively, and his Ph.D. from Zhejiang University, Zhejiang, China, in 1993. He was with the Central South University, Changsha, China, as a Professor from 1996 to 2002. Starting in 2003, he became a Professor at 
Hunan University. His current research interests include harmonics power conversion systems, harmonics suppression, reactive power compensation, and electric power savings. He has published over 100 journal and conference articles. He was a recipient of the 2006 National Scientific and Technological Awards of China, the 2005 Scientific and Technological Awards from the National Mechanical Industry Association of China and the 2007 Scientific and Technological Awards from the Hunan Province of China. He is currently serving as the Associate Board Chairperson of the Hunan Society of Electrical Engineering. He is also serving as the Chief of Hunan Electric Science and Application Laboratory. 